\title{
DESEMPENHO REPRODUTIVO DE NOVILHAS DE CORTE EXPOSTAS A DIFERENTES MÉTODOS DE BIOESTIMULAÇÃO
}

\author{
REPRODUCTIVE PERFORMANCE OF BEEF HEIFERS EXPOSED TO DIFFERENT \\ BIOESTIMULATIONTREATMENTS
}

\author{
Menezes, L.M. ${ }^{1,3}$, Brauner, C.C. ${ }^{1}$, Pimentel, M.A. ${ }^{1}$, Moraes, J.C.F. ${ }^{2}$ e Amaral, F.A. ${ }^{1}$ \\ ${ }^{1}$ Departamento de Zootecnia. Faculdade de Agronomia Eliseu Maciel. Universidade Federal de Pelotas. \\ Pelotas-RS. Brasil. menezes@veterinario.med.br \\ Embrapa Centro de Pesquisa Pecuária Sul. Bagé. Brasil. ferrugem@cppsul.embrapa.br \\ ${ }^{3}$ Campus de Ciências Rurais. Universidade da Região da Campanha. Bagé. Brasil.
}

\author{
PaLAVRAS ChAVE ADICIONAIS \\ Concepção. Índice de prenhez.
}

\section{RESUMO}

O objetivo deste estudo foi avaliar o desempenho reprodutivo de novilhas de corte submetidas a diferentes métodos de bioestimulação. Foram utilizadas 220 novilhas Aberdeen Angus de dois anos de idade. Setenta e três novilhas foram mantidas com machos caudectomizados, 73 com vacas androgenizadas e 74 não receberam tratamento bioestimulatório. Os tratamentos tiveram duração de 60 dias préacasalamento. Os fatores fixos analisados foram os três tratamentos. As variáveis resposta foram os pesos ao início e fim dos tratamentos, ganho médio diário (GMD), o índice de prenhez e dias para a concepção (medido através da data de parto ajustada a data de inseminação). Não houve diferença $(p>0,05)$ nos índices de prenhez obtidos entre os tratamentos. A concepção foi antecipada $(p<0,01)$ no grupo bioestimulado por vacas androgenizadas, quando comparada ao grupo exposto a machos caudectomizados. A bioestimulação não é capaz de alterar os índices de prenhez em novilhas de corte. A utilização da bioestimulação com vacas androgenizadas antecipa a concepção em novilhas de corte de dois anos de idade.

\section{SUMMARY}

The objective of this study was to evaluate the reproductive performance of beef heifers exposed or not to different sexual biostimulation treatments. Two hundred and twenty Aberdeen Angus heifers,

Recibido: 26-10-10. Aceptado: 13-4-11.

\author{
Additional KEYWORDS \\ Conception. Pregnancy rate.
}

24 months old were used. Heifers were divided into three groups: group one, using teaser bull consisted of 73 heifers exposed, for 60 days previous to $\mathrm{Al}$, to vasectomized males. The second group consisted of 73 heifers exposed during the same period to androgenized cows. The third group consisting of 74 heifers was the control group. After the treatments (60 days) started the breeding season. Variables analyzed were the weight at the beginning and the end of treatment, $A D G$, the pregnancy rate, and the interval between the start of breeding season and conception. Fixed factors considered were the three treatments. Heifers biostimulatated with androgenized cows had a earlier conception, $(p<0.01)$ than thosed bioestimulated with teaser bull. Biostimulation can not change rates of pregnancy in beef heifers. The use of biostimulation with cows androgenized anticipates the conception in beef heifers of two years old.

\section{INTRODUÇÃO}

A existência de aspectos sociais dentro dos rebanhos é cientificamente comprovada, no qual o grau de interação de diferentes animais pode ativar o sistema neuroendócrino, estimulando ou inibindo a reprodução. Um tipo de interação que ocorre é caracterizada pelo fenômeno conhecido 
como efeito macho ou bioestimulação, que Chenoweth (1983) define como o estímulo produzido pelo macho capaz de alterar processos físicos e comportamentais de fêmeas de mesma espécie.

Em bovinos, parece haver menos estudo sobre este fenômeno do que em outras espécies. Entretanto, um maior esclarecimento sobre tal poderia ser importante como estratégia mercadológica (Menezes et al., 2010), uma vez que os mercados que melhor remuneram o produto carne bovina têm apontado uma tendência por produtos limpos, livres de resíduos de hormônios e que sejam ambientalmente corretos. Assim, a utilização da bioestimulação poderá ser uma ferramenta a se considerar como alternativa de manejo.

Utilizando diversas técnicas de bioestimulação, alguns autores têm demonstrado melhora no desempenho reprodutivo de novilhas de corte (Assis et al., 2000), enquanto outros não encontraram diferenças (Berardinelli et al.,1978). O objetivo deste estudo foi avaliar o desempenho reprodutivo de novilhas de corte expostas a diferentes métodos de bioestimulação.

\section{MATERIAISEMÉTODOS}

Foi avaliado o desempenho reprodutivo de 220 novilhas de corte da raça Aberdeen Angus, com idade média inicial entre 21 e 24 meses de idade, apresentando escala de condição corporal (ECC) $\geq 3$, numa escala de 1 a 5 . O exame ginecológico foi realizado com auxílio de aparelho de ultrassom (Aloka 210 $\mathrm{x}$; transdutor de $5 \mathrm{MHz}$ ). Foram consideradas cíclicas todas as novilhas que apresentaram folículos superiores a $10 \mathrm{~mm}$ e tônus uterino (T.U.) caracterizado como 2 ou 3 , em uma escala de 1-3, ou ainda, presença de corpo lúteo. Após este exame, as novilhas foram pesadas e separadas aleatoriamente em três grupos: O primeiro grupo, composto por 73 novilhas foi mantido durante 60 dias pré-acasalamento com seis machos rufiões (novilhos caudectomizados bilateralmente).
O segundo, composto por 73 novilhas foi mantido pelo mesmo período com seis vacas adultas androgenizadas, através da aplicação de testosterona (ciclopropionato de testosterona) na dose de $1 \mathrm{mg} / \mathrm{kg}$. O terceiro grupo, denominado controle foi composto por 74 animais mantidos isolados. Os potreiros eram distantes 600 metros entre si, e ao término dos 60 dias todas as novilhas passaram a fazer parte de um único grupo e iniciou-se a estação de acasalamento, realizada através de inseminação artificial convencional (IA) por 45 dias.

A estação reprodutiva foi complementada com mais 45 dias de monta natural, utilizando-se touros na proporção de 1:25 novilhas. Após 60 dias do término da estação de acasalamento foi realizado o diagnóstico de gestação, através de palpação retal. Controlou-se a data de parto, sendo assim possível calcular o tempo necessário (em dias) entre o início do acasalamento até a concepção (IAC). A partir da data de parto, regrediu-se 283 dias (tempo de gestação fisiológico da raça), e em seguida, ajustando-se para a data da inseminação. Para as datas de parto fora do período de inseminação foram considerados apenas os 283 dias no momento do cálculo da data de concepção (por monta natural).

Os animais foram pesados após jejum prévio de 12 horas em duas oportunidades: após o exame ginecológico, no momento da formação dos grupos e início dos tratamentos bioestimulatórios (PITB) e ao início da IA, após 60 dias de tratamentos (PATB). Os fatores fixos utilizados foram os três tratamentos. As variáveis respostas foram as taxas de gestação, tempo necessário (em dias) do início do acasalamento até a concepção (IAC) e o peso vivo, além do ganho médio diário(GMD). A freqüência de novilhas dentro dos tratamentos e dentro do fator taxa de gestação foi analisada através do teste de Qui-quadrado. A variação entre os dias necessários para concepção (IAC), peso vivo e ganho médio diário das novilhas foi analisada através da 
Tabela I. Análise de freqüência para o fator gestação, peso vivo ao início e fim da bioestimulação e ganho médio diário de peso vivo para os respectivos tratamentos. (Frequency analysis for the factor gestation, body weight at the beginning and end of the biostimulation and average daily gainweight for the respective treatments).

\begin{tabular}{lccccr}
\hline & \multicolumn{2}{c}{ Gestação $(\%)$} & \multicolumn{2}{c}{ Peso vivo $(\mathrm{kg})$} & \\
& Prenha & Vazia & Início (11/10) & Fim (15/12) & GMD \\
\hline Macho (73) & $84,9(62)$ & $15,1(11)$ & $276,1 \pm 2,6$ & $341,1 \pm 2,9$ & 1,160 \\
Vaca (73) & $84,9(62)$ & $15,1(11)$ & $273,8 \pm 2,6$ & $349,1 \pm 2,9$ & 1,340 \\
Testemunha (74) & $83,8(62)$ & $16,2(12)$ & $269,7 \pm 2,6$ & $342,5 \pm 2,9$ & 1,310 \\
Geral (220) & $84,6(186)$ & $15,4(34)$ & 273,250 & 344,280 & 1,270 \\
& & & & &
\end{tabular}

$\chi^{2}=0,049510, G L=2, p=0,97$.

análise de variância (ANOVA), considerando o seguinte modelo:

$$
Y_{i j}=\mu+B M_{i}+B V_{j}+e_{i j}
$$

Emque:

$Y_{i j}=$ uma observação de IAC, PITB, PATB e GMD, $\mu=$ média geral,

i= bioestimuladas com machos e não bioestimuladas,

$\mathrm{j}=$ bioestimuladas com vacas androgenizadas e não bioestimuladas e

$e_{i j}=$ erro experimental.

Para análise dos dados foi utilizado o programa NCSS 7.0(2007).

\section{RESULTADOSEDISCUSSÃO}

Não houve diferença entre as taxas de gestação dos distintos tratamentos $(p>0,05)$. Os valores, em percentual de novilhas prenhes podem ser visualizados na tabela $\mathbf{I}$. Os altos índices de prenhez obtidos podem ser explicados pelo alto nível nutricional a que as novilhas estavam submetidas. $\mathrm{O}$ peso médio ao início do acasalamento $(344,4$ $\mathrm{kg}$ ) é muito superior ao peso limite citado por Pimentel et al. (1981), que trabalhando com novilhas criadas no Rio Grande do Sul em condições extensivas verificaram um índice de prenhez significativamente maior em novilhas com peso corporal igual ou superior a $270 \mathrm{~kg}$, indicando este peso como base para o início do período reprodutivo.
Ainda na tabela I, pode-se verificar que as novilhas não apresentaram diferenças de peso corporal, entre os tratamentos, do início até o fim do período experimental $(\mathrm{p}>0,05)$.

De acordo com a tabela II, verifica-se que praticamente todas as novilhas utilizadas já estavam cíclicas ao início dos tratamentos, de acordo com os critérios utilizados. Tal fato também pode ser um dos fatores causais do alto índice de prenhez percebido.

Em relação ao tempo necessário para concepção (em dias) os tratamentos demonstraram efeito $(\mathrm{p}<0,05)$, de acordo com a tabela III. Novilhas bioestimuladas por vacas androgenizadas conceberam em

Tabela II. Condição ginecológica das novilhas avaliadas por ultrassonografia ao início do período experimental, de acordo com respectivo tratamento. (Gynecologic condition of heifers evaluated by sonography at the beginning of the trial period, according to treatment).

Corpo Folículo $\geq 10 \mathrm{~mm}$ Imatura lúteo T.U. entre 2-3

\begin{tabular}{lccc}
\hline Macho (73) & 52 & 21 & 0 \\
Vaca (73) & 55 & 18 & 0 \\
Testemunha (74) & 65 & 8 & 1 \\
Geral (220) & 172 & 47 & 1 \\
\hline
\end{tabular}


média 7 dias antes que novilhas bioestimuladas por rufiões caudectomizados. Makarechian et al. (1985) encontraram resultado semelhante, utilizando touros como ferramenta bioestimulatória. Tais autores verificaram antecipação na data de parto na ordem de 5,5 dias, em comparação com um grupo controle. Apesar da diferença numérica poder ser considerada baixa, a antecipação da concepção e consequentemente do parto deve ser uma meta constante em um estabelecimento que se dedica a cria de bovinos de corte. Tal antecipação possibilita ainda, o encurtamento do período de acasalamento, que de acordo com Short et al. (1990) é a principal estratégia de manejo a ser realizada quando se objetiva viabilizar maiores índices de prenhez no acasalamento subseqüente.

A otimização do índice de repetição de prenhez na fêmea primípara passa inevitavelmente pela concepção precoce quando ainda novilha. A antecipação da primeira concepção reduz ou mesmo anula a possibilidade de haver fêmeas em anestro quando a estação de acasalamento subseqüente iniciar, o que é fundamental quando

\section{BIBLIOGRAFIA}

Assis, R.R., Pimentel, M.A., Jardim, P.O., Osório, J.C.S. e Machado, J.P.M. 2000. Influência da bioestimulação com machos vasectomizados na eficiência reprodutiva de novilhas Aberdeen Angus. Rev. Bras. Agrociênc., 6: 226-231.

Berardinelli, J.G., Fogwell, R.L. and Inskeep, E.K. 1978. Effect of electrical stimulation or presence of a bull on puberty in beef heifers. Theriogenology, 9: 133

Chenoweth, P.J. 1983. Reproductive management procedures in control of breeding. Anim. Prod. Aust., 15: 28.

Makarechian, M., Farid, A. and Berg, R.T. 1985. Effect of exposure to bulls and body weight on reproductive performance in beef heifers. Can. J. Anim. Sci., 65: 31.
Tabela III. Médias ajustadas e erro padrão da média do intervalo entre o início do acasalamento e a concepção (em dias) para os diferentes tratamentos. (Adjusted means and standard error of the mean of interval between the onset of breeding and conception (in days) for the different treatments).

\begin{tabular}{lc}
\hline & $\begin{array}{c}\text { Intervalo (dias) } \\
\text { acasalamento-concepção }\end{array}$ \\
Macho (62) & $19,9 \pm 1,7^{\mathrm{a}}$ \\
Vaca (62) & $13,0 \pm 1,6^{\mathrm{b}}$ \\
Testemunha (62) & $18,2 \pm 1,6^{\mathrm{ab}}$ \\
Geral (186) & 17,0
\end{tabular}

abMédias seguidas de letras distintas na coluna diferem entre si $(p<0,05)$.

se objetiva um terneiro/vaca/ano.

\section{CONCLUSÃO}

A bioestimulação não alterou o índice de prenhez de novilhas de dois anos de idade. A utilização de bioestimulação com vacas androgenizadas antecipou a concepção de novilhas de corte, sendo preferível a utilização de machos caudectomizados.

Menezes, L.M., Brauner, C.C. e Pimentel, M.A. 2010. Efeitos da bioestimulação sobre a performance reprodutiva em bovinos de corte. Arch. Zootec., 59: 1-13.

NCSS 7.0. 2007. Statistical System for Windows. User's Guide I, II, III. Kaysville. Utah.

Pimentel, C.A., Cardelino, R.A. e Oliveira, J.A.F. 1981. Peso corporal de novilhas de corte, como referência para o acasalamento. Anais da XVIII Reunião da SBZ. Sociedade Brasileira de Zootecnia. Goiânia. pp. 261.

Short, R.E., Bellows, R.A., Staigmiller, R.B., Berardinelli, J.G. and Custer, E.E. 1990. Physiological mechanisms controlling anestrus and infertility in pospartum beef cattle. J. Anim. Sci., 68: 799-815. 